\title{
Meta-analysis
}

\section{e Comparison of 7 Surgical Interventions for Lumbar Disc Herniation: A Network Meta-analysis}

Fan Feng, MD ${ }^{1,2}$, Qianqian Xu, MD², Feifei Yan, MD², Yuanlong Xie, MD², Zhouming Deng, $\mathrm{MD}^{2}$, Chao $\mathrm{Hu}, \mathrm{MD}^{2}$, Xiaobin Zhu, MD², and Lin Cai, MD²

From: ${ }^{2}$ Department of Pain Management, The Central Hospital of Wuhan, Tongji Medical College, Huazhong

University of Science and

Technology, China; ${ }^{2}$ Wuhan University Zhongnan Hospital,

China

Address Correspondence: Lin Cai, MD Wuhan University Zhongnan

Hospital

169 Donghu Rd

Wuchang Qu, Wuhan Shi

Hubei Sheng, China 430071

E-mail:

orthopedics@whu.edu.cn

Disclaimer: There was no external funding in the preparation of this manuscript. Conflict of interest: Each author certifies that he or she, or a member of his or her immediate

family, has no commercial association (i.e., consultancies, stock ownership, equity interest, patent/licensing arrangements, etc.) that might pose a conflict of interest in connection with the submitted manuscript.

Manuscript received: 02-25-2016 Revised manuscript received: 02-27-2017

Accepted for publication: 03-09-2017

Free full manuscript: www.painphysicianjournal.com
Background: The number of interventions on intervertebral discs rapidly increased and the treatment options for lumbar disc surgery quickly evolved. It is important that the safety and efficacy of all new innovative procedures be compared with currently accepted forms of treatment; however, the previous pairwise meta-analyses could not develop the hierarchy of these treatments.

Objectives: The purpose of the study is to perform a network meta-analysis to evaluate the clinical results of 7 surgical interventions for the treatment of lumbar disc herniation.

Study Design: Network meta-analysis of randomized controlled trials (RCTs) for multiple treatment comparisons of lumbar disc herniation.

Methods: We performed a Bayesian-framework network meta-analysis of RCTs to compare 7 surgical interventions for people with lumbar disc herniation. The eligible RCTs were identified by searching Embase, Pubmed, the Cochrane Central Register of Controlled Trials (CENTRAL), and Google scholar. Data from 3 outcomes (success, complications, and reoperation rate) were independently extracted by 2 authors.

Results: A total of 29 RCTs including 3,146 participants were finally included into this article. Our meta-analysis provides hierarchies of these 7 interventions. For the success rate the rank probability (from best to worst): percutaneous endoscopic lumber discectomy (PELD) > standard open discectomy (SOD) > standard open microsurgical discectomy (SOMD) > chemonucleolysis $(\mathrm{CN})>$ microendoscopic discectomy (MED) > percutaneous laser disc decompression (PLDD) > automated percutaneous lumber discectomy (APLD). For the complication rate the rank probability (from best to worst): PELD > SOMD > SOD > MED > PLDD > CN > APLD. For the reoperation rate the rank probability (from best to worst): SOMD > SOD > MED > PLDD > PELD > CN $>$ APLD.

Limitations: The limitations of this network meta-analysis include the range of study populations and inconformity of the follow-up times and outcome measurements

Conclusions: This meta-analysis provides evidence that PELD might be the best choice to increase the success rate and decrease the complication rate, moreover SOMD might be the best option to drop the reoperation rate. APLD might lead to the lowest success rate and the highest complication and reoperation rate. Higher quality RCTs and direct head to head trials are needed to confirm these results.

Key words: Lumbar disc herniation, discectomy, minimally invasive surgery, network meta-analysis

Pain Physician 2017; 20:E863-E871 ciatica, which is caused by nerve root compression or irritation, includes the symptoms of leg pain and occasionally neurological disturbance in the dermatome of the affected nerve root. Over $90 \%$ of cases are due to symptomatic lumbar disc herniation (LDH) (1). Symptomatic LDH, with a reported prevalence of $1 \%-3 \%(2)$, is the most common pathological process leading to spinal surgery $(3,4)$. 
The surgical approaches include open discectomy and a wide variety of minimally invasive techniques (5).

In 1934, Mixter and Barr were the first authors to treat symptomatic LDH surgically by performing an open laminectomy and discectomy (6), which has been regarded as a "standard" surgical procedure $(3,5)$. The standard open discectomy (SOD) technique could relieve patients' pain and improve their nerve function. However, the greatest problem is the surgical trauma of paravertebral muscles, which is related to failed back surgery syndrome $(1,7)$.

Since then the number of interventions on intervertebral discs rapidly increased and the treatment options for lumbar disc surgery quickly evolved. The surgical procedures changed over time and were continuously being refined. In the late 1960s, the surgical microscope was introduced for spinal surgery by Mahmut Gazi Yasargil (8) and his colleague Wolfhard Caspar (9), and so-called standard open microsurgical discectomy (SOMD) was introduced (10). Foley (11) introduced the microendoscopic discectomy (MED) technique in 1997. This minimally invasive technique was performed by a transmuscular approach with advanced optics (11). Besides open discectomy, other interventional techniques were developed to overcome the side effects of surgical procedures. In 1964 the American orthopedic surgeon, Lyman Smith (12) introduced chemonucleolysis (CN), a minimally invasive technique consisting only of a can- nula and the proteolytic enzyme chymopapain, which is injected into the disc compartment to dissolve the displaced disc material. In 1975 the Japanese orthopedic surgeon Sadahisa Hijikata et al (13) first reported the development of percutaneous techniques for the treatment of certain types of lumber disc herniations. In 1985, Onik et al (14) reported the use of what has come to be called automated percutaneous lumber discectomy (APLD). Further variants of minimally invasive surgical procedures, such as percutaneous laser disc decompression (PLDD) in 1986 and percutaneous endoscopic lumber discectomy (PELD) in 1990s, were also introduced $(15,16)$. Of the techniques available, open discectomy, performed with (micro-) or without the use of an operating microscope, is the most common, but those less invasive surgical techniques have gained popularity in recent years. Figure 1 shows the equipment used in a variety of surgical procedures.

It is particularly important that the safety and efficacy of all new innovative procedures be compared with currently accepted forms of treatment. Previous pairwise meta-analyses could not develop the hierarchy of these treatments because some treatments had not been compared one by one (17). In addition, the number of included randomized controlled trials (RCTs) was limited, which led to some potential incorrect conclusions. We aimed to compare the clinical results of 7 surgical interventions (SOD, SOMD, MED, CN, PLLD, APLD,
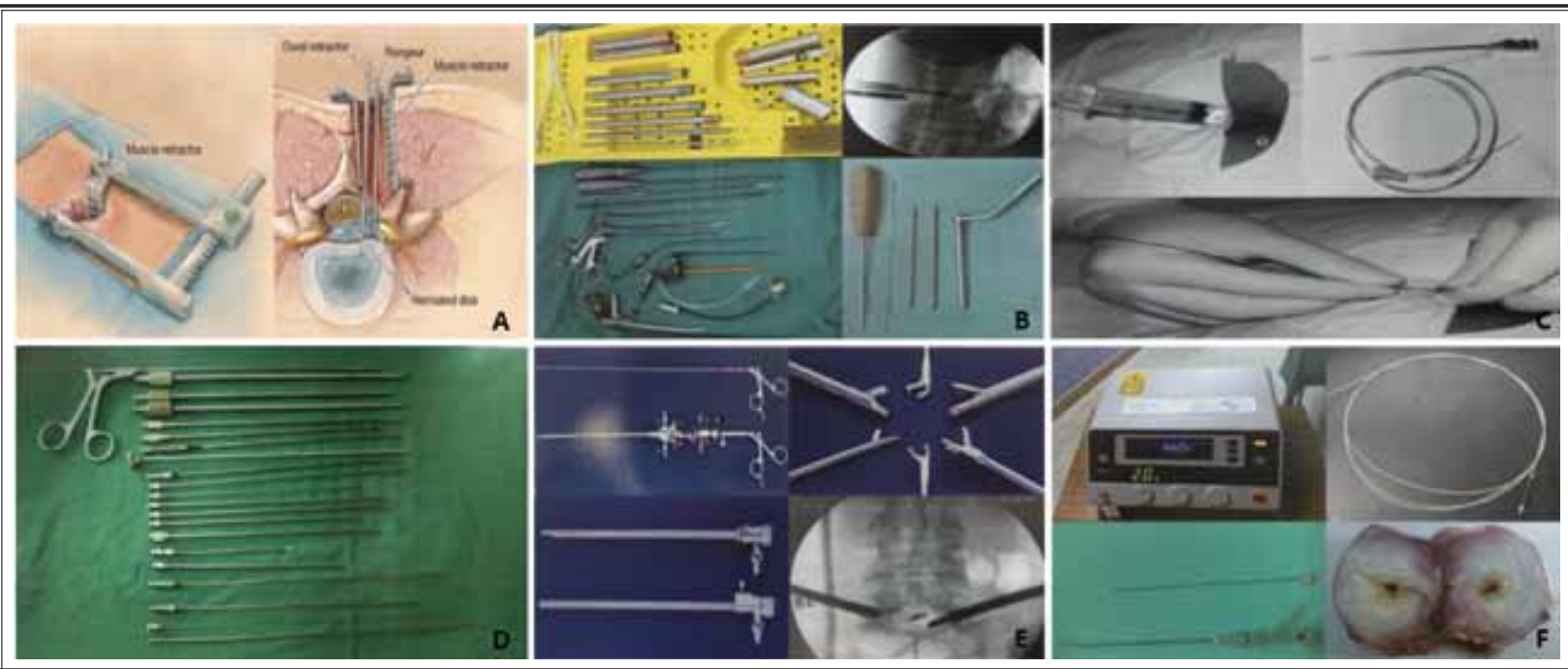

Fig. 1. Equipment for different surgical procedures. Fig. 1A. Instrumentation for SOMD; Fig. 1B. Instrumentation for MED; Fig. 1C. Instrumentation for CN; Fig. 1D. Instrumentation for APLD; Fig. 1E. Instrumentation for PELD; Fig. 1F. Instrumentation for PLDD. 
and PELD) for the treatment of LDH and to provide a hierarchy of the comparative success rate, complication rate, and reoperation rate.

\section{Methods}

\section{Criteria for Considering Studies}

We only included RCTs, which compared ratings of success and incidences of complications and reoperations of the 7 interventions (SOD, SOMD, MED, CN, PLLD, APLD, and PELD) in people with LDH.

Studies were included if they met the following criteria: (1) Study design: RCTs; (2) Participants: patients with LDH who have indications for surgical intervention; (3) Interventions and comparisons: therapy that included 2 of the 7 interventions; (4) Outcomes: the studies reported rates of success and incidences of complications and reoperations (to pool the results, ratings of excellent, good, and fair were classified as "success" and poor, unimproved, and worse as "failure").

Trials were excluded if: (1) they were abstracts, letters, or meeting proceedings; (2) they contained repeated data or did not report the outcomes of interest; and (3) the duration of follow-up was $<6$ months.

\section{Search Methods and Study Selection}

We searched EMBASE (from 1974 to May 2016), PubMed (from 1966 to May 2016), the Cochrane Central Register of Controlled Trials (CENTRAL) (The Cochrane Library, most recent issue), and Google scholar. Key words and MeSH terms including "lumbar disc herniation," "open discectomy," "microsurgery," "minimally invasive surgery," and "percutaneous discectomy" were used in the search strategy. We also viewed the reference lists of the included studies for any additional papers. We included only articles written in English.

Two authors independently made the selection based on the title and abstract. Any disagreement between the 2 authors was resolved by discussion. If there was no consensus, a third reviewer (Yuanlong Xie) was consulted.

\section{Data Collection and quality Assessment}

We used the Cochrane risk of bias tool to assess risk bias of included studies (18). The tool has 7 domains including random sequence generation, allocation concealment, blinding of participants and experimenters, blinding of outcome assessment, incomplete outcome data, selective reporting, and other bias. Since it was sometimes difficult to blind surgeons and patients, we did not include the blinding items of risk of bias in our analysis. The classification of the judgment for each domain was low risk of bias, high risk of bias, or unclear risk of bias, and 2 authors independently evaluated the risk of the studies.

\section{Data Synthesis and Analysis}

We used pairwise meta-analyses for studies which directly compared different treatments by using Stata software (version 12.0, StataCorp, College Station, TX). DerSimonian and Laird random effects model was used. The pooled estimates of odds ratios (ORs) and $95 \%$ confidence intervals $(\mathrm{Cl})$ of 3 outcomes were shown. Chi-square test and 12 test were used for testing heterogeneity among the studies. Then network meta-analysis was performed by using WinBUGS (version 1.4.3, MRC Biostatistics Unit, Cambridge, UK) with random effects models developed by Chaimani (downloaded from www.mtm.uoi.gr) (19). We used the Markov Chains Monte Carlo (MCMC) method to get results, which were reported as posterior distribution median with $95 \% \mathrm{Cl}$. Non-informative uniform and normal prior distributions were performed to fit the model (20). An automatically generated starting value was used to fit the model. For each analysis, we used 100,000 iterations after an initial burn-in of 5,000 (21). To rank the treatments, we used 2 ways. Firstly, we used posterior probabilities of outcomes to calculate probabilities of treatment ranking. Secondly, we used the surface under the cumulative ranking probabilities (SUCRA) to indicate which treatment was the best one (22).

The funnel plot was used to identify possible publication bias if the number of included studies in one comparison was larger than 10 . The sensitivity analysis was performed by excluding studies with different durations of follow-up and studies with high risk of bias. The Cochrane review protocol was not required for this research.

\section{Results}

The PRISMA flow diagram of study selection is depicted in Fig. 2. The search was performed on April 10,2016 , and 1,025 references were identified in the primary search and 3 through other sources. After removal of duplicates, irrelevant studies, case reports, and studies that were not comparative studies, 72 records were screened. Twenty-nine studies with 31 published articles were eligible for inclusion, and others were not selected for various reasons. Data from these studies 

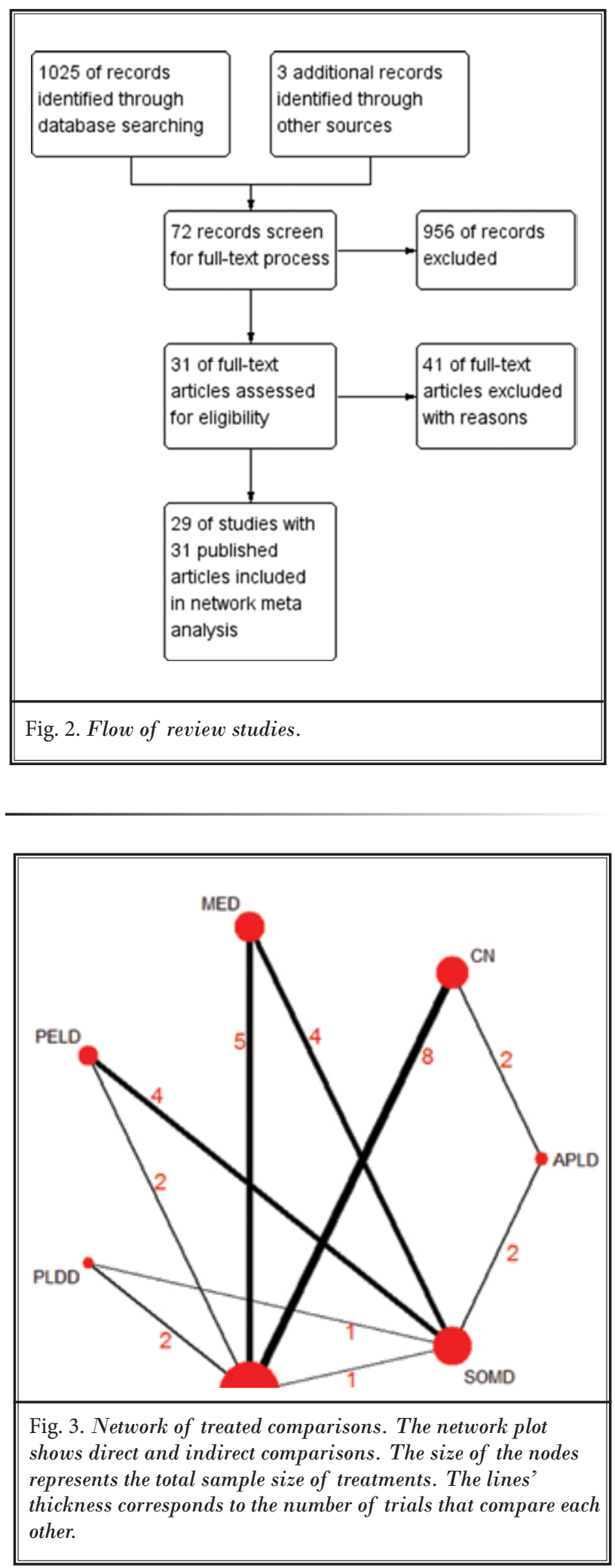

were included in the meta-analysis (23-53). Figure 3 shows the network of treated comparisons.

Table 1 provides a summary of the studies included in the review. A total of 3,146 participants were included in this meta-analysis. The study sample size ranged from 20 to 358. These studies were published between 1983 and 2016.

\section{Risk of Bias in Included Studies}

Figure 4 shows the risk of bias in all 29 studies. Two studies had a high risk of bias for sequence allocation and concealment because they were reported as openlabel clinical trials $(30,39)$. Since it was sometimes difficult to blind surgeons and patients, we did not include the blinding items for risk of bias in our analysis.

\section{Success Rate-related Outcomes}

A total of 762 patients were assigned to SOD therapy, 237 to SOMD therapy, 468 to CN therapy, 250 to MED therapy, 219 to PELD therapy, 41 to APLD therapy, and 132 to PLLD therapy.

Compared with SOD therapy, PELD (OR 1.47, 95\% $\mathrm{Cl} 0.45$ - 3.70) yielded the most significant effect on success rate, followed by CN (OR 0.78, $0.44-1.26)$, SOMD (OR 0.77, $0.19-2.18$ ), PLDD (OR 0.49, $0.15-1.25$ ), and APLD (OR 0.14, $0.02-0.55$ ). Details pertaining to other comparisons are listed Table 2.

\section{Complication Rate-related Outcomes}

A total of 460 patients were assigned to SOD therapy, 461 to SOMD therapy, 188 to CN therapy, 456 to MED therapy, 221 to PELD therapy, 69 to APLD therapy, and 63 to PLLD therapy.

Compared with SOD therapy, APLD (OR 172, 95\% Cl 0.27 - 401) had the most significant effect on complication rate, followed by CN (OR 76.16, $0.31-174)$, PLDD (OR 2.52, 0.20 - 10.41), MED (OR 2.29, 0.98 - 4.78), SOMD (OR 1.78, 0.65 - 4.24), and PELD (OR 0.37, 0.09 0.98 ). Details pertaining to other comparisons are listed Table 3.

\section{Reoperation Rate-related Outcomes}

A total of 559 patients were assigned to SOD therapy, 551 to SOMD therapy, 313 to CN therapy, 456 to MED therapy, 223 to PELD therapy, 110 to APLD therapy, and 153 to PLLD therapy.

Compared with SOD therapy, APLD (OR 46.75, 95\% CI 2.78 - 233) had the most significant effect on complication rate, followed by CN (OR 15.89, $4.07-47.53$ ), 


\begin{tabular}{|c|c|c|c|c|c|c|c|c|c|c|c|c|c|c|c|c|c|c|c|c|c|c|c|c|c|c|}
\hline 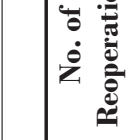 & $\frac{\infty}{1}$ & $\underset{\Sigma}{\Xi}$ & 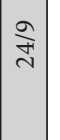 & $\frac{\pi}{m}$ & $\vec{े}$ & $\vec{\Xi}$ & $\stackrel{\circ}{\infty}$ & $\stackrel{n}{\sim}$ & & $\overleftrightarrow{z} \mid$ & $\stackrel{\Sigma}{\prime}$ & $\overleftrightarrow{z}$ & $\hat{\sigma}) \stackrel{N}{=}$ & $\overleftrightarrow{z}$ & $\stackrel{?}{=}$ & $\frac{\rho}{m}$ & $\bar{a}$ & $\mathbb{z}$ 岁 & $\vec{m}$ & $\Xi \frac{\circ}{1}$ & 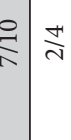 & $\vec{\lambda}$ & $\frac{\sqrt{m}}{\infty}$ & $\equiv \infty$ & $\begin{array}{c}\frac{1}{\infty} \\
-\infty \\
-\infty\end{array}$ & \\
\hline 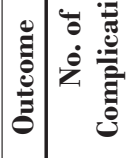 & $\overleftrightarrow{z}$ & 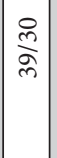 & $\frac{i n}{m}$ & $\overleftrightarrow{z}$ & $\mathbb{z}$ & $\stackrel{?}{=}$ & $\stackrel{\circ}{\circ}$ & $\stackrel{m}{\sim}$ & $\stackrel{ \pm}{\Sigma}$ & $\mathbb{z}$ & $\stackrel{s}{\delta}$ & $\overleftrightarrow{z}$ & $\underset{-\infty}{\infty} \mid \overleftrightarrow{z}$ & $\mathbb{z}$ & $\overleftrightarrow{z}$ & $\vec{z}$ & $\stackrel{\ominus}{=}$ & $\frac{7}{\pi}$ & $\frac{N}{\mathrm{in}}$ & $\vec{m} \frac{\tilde{m}}{\mathrm{~m}}$ & 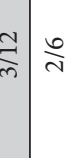 & $\overleftrightarrow{z}$ & $\begin{array}{l}\stackrel{n}{0} \\
\stackrel{2}{2} \\
\frac{a}{-}\end{array}$ & $\vec{m}$ & $\vec{z}=$ & \\
\hline 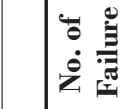 & $\overleftrightarrow{z}$ & $\frac{\vec{m}}{\hat{q}}$ & 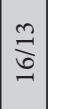 & $\mathbb{z}$ & 交 & $\stackrel{m}{\stackrel{m}{-}}$ & $\mathrm{s}$ & $\mathbb{z}$ & $\stackrel{m}{\sim}$ & $\stackrel{n}{N}$ & $\stackrel{\frac{1}{\infty}}{=}$ & $\Xi$ & $\overleftrightarrow{z} \overleftrightarrow{z}$ & $\underset{m}{\infty}$ & $\stackrel{\mathrm{S}}{=}$ & $\frac{m}{m}$ & 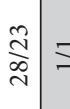 & $=\frac{m}{\lambda}$ & 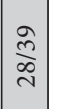 & 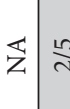 & $\stackrel{n}{a} \mid \mathbb{z}$ & $\frac{F}{m}$ & $\vec{z}$ & $\stackrel{m}{F}$ & & \\
\hline 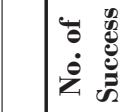 & $\overleftrightarrow{z}$ & 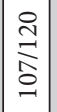 & $\underset{m}{\vec{\sigma}}$ & $\overleftrightarrow{z} \cong$ & సै & 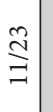 & $\stackrel{m}{ \pm}$ & $\overleftrightarrow{z}$ & 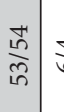 & $\frac{H}{6}$ & 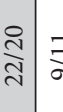 & $\vec{\Xi}$ & $\mathbb{z} \mathbb{z}$ & $\underset{\substack{7 \\
D}}{\mathcal{F}}$ & 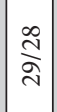 & $\underset{ }{\stackrel{1}{二}}$ & $\underset{\substack{\tilde{\infty} \\
\sim}}{\stackrel{\overbrace{}}{\sigma}}$ & רֵ & 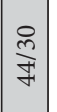 & $\overleftrightarrow{z} \mid \frac{\infty}{a}$ & \begin{tabular}{l|l}
$\infty$ & $\mathbb{2}$ \\
& \\
$\infty$
\end{tabular} & $\frac{\mathrm{O}}{\mathrm{i}}$ & $\overleftrightarrow{z}$ & 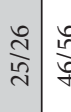 & & \\
\hline 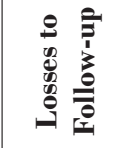 & 0 & $\vec{\sim}$ & $\infty$ & $\circ 0$ & 0 & $\sim$ & $\overleftrightarrow{z}$ & 0 & \begin{tabular}{l|l}
0 & 1
\end{tabular} & $\wedge$ & 00 & 0. & $\therefore 0$ & 0 & 0 & 0 & 㐏 & 0 & $\stackrel{\sim}{\sim}$ & $0 \approx$ & \begin{tabular}{c|c}
$*$ & 0
\end{tabular} & 0 & $\stackrel{\infty}{\sim}$ & $N$ & 0 & \\
\hline 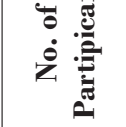 & ¿্ণ & $\underset{m}{\infty}$ & $\stackrel{20}{=}$ & of & $\pi$ & in & ते & ¿ & $\Xi$ & $\stackrel{m}{m}$ & $8 \approx$ & ה & 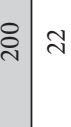 & $\stackrel{\infty}{\infty}$ & 8 & 와 & $\alpha$ & 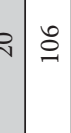 & 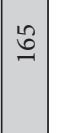 & \& & 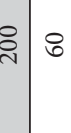 & 8 & 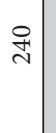 & 8 & $\vec{a}$ & \\
\hline 产 & - & $\rightarrow$ & - & -14 & . & $\neg$ & -1 & -1 & $-t$ & ? & \begin{tabular}{lll}
$\stackrel{0}{i}$ & \multicolumn{1}{c}{}
\end{tabular} & • & \begin{tabular}{l|l}
0 & $N$
\end{tabular} & $\sim$ & $\hat{i}$ & $\sim$ & -12 & $\stackrel{8}{\delta}-7$ & -7 & $\sim \sim$ & $\sim \underset{\rightarrow}{\sim}$ & $\overleftrightarrow{z}$ & $\sim$ & - & - & \\
\hline 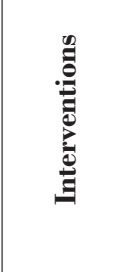 & 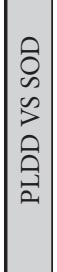 & 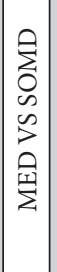 & $\begin{array}{l}0 \\
0 \\
0 \\
\infty \\
s \\
0 \\
0 \\
\vdots \\
\vdots\end{array}$ & $\begin{array}{l}0 \\
0 \\
\infty \\
\infty \\
> \\
z \\
z \\
z\end{array}$ & 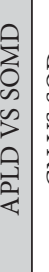 & $\begin{array}{l}0 \\
0 \\
0 \\
s \\
z \\
z\end{array}$ & $\begin{array}{ll}0 & \\
0 & \\
\infty & \\
& \\
z & \end{array}$ & 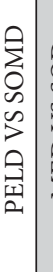 & 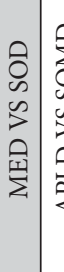 & 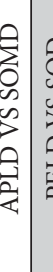 & 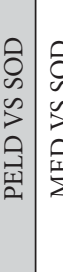 & 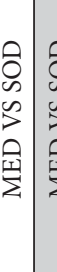 & 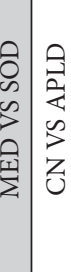 & $\begin{array}{l}0 \\
0 \\
0 \\
z \\
z \\
z\end{array}$ & 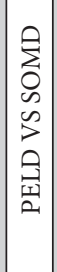 & 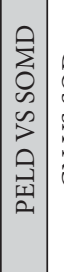 & 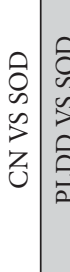 & 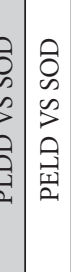 & 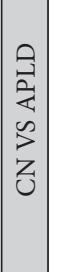 & 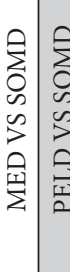 & 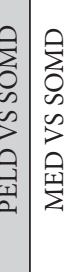 & $\begin{array}{l}0 \\
0 \\
\infty \\
z \\
z \\
0\end{array}$ & 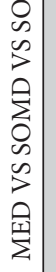 & 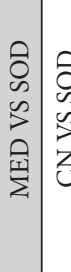 & 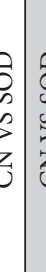 & \\
\hline 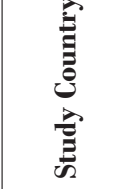 & 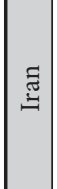 & 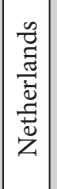 & 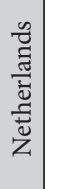 & 光 & है & 岇 & 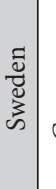 & 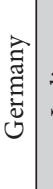 & $\stackrel{\dddot{g}}{\Xi}$ & 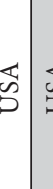 & کૅ & 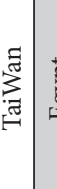 & 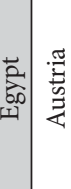 & 营 & 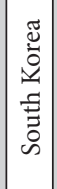 & 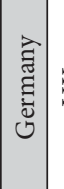 & $\breve{s}$ & ปี & 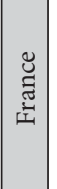 & 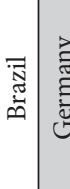 & 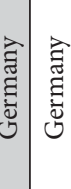 & 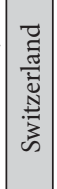 & 氙 & 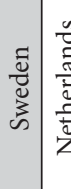 & & \\
\hline 胥 & 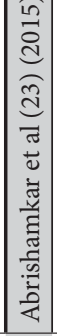 & 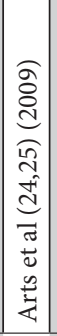 & 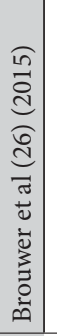 & 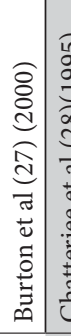 & 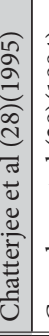 & 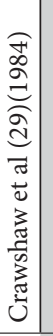 & 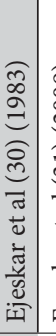 & 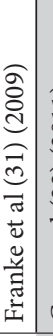 & 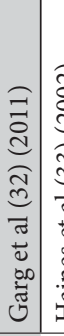 & 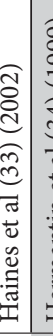 & 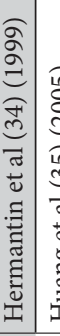 & 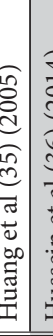 & 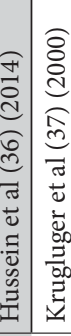 & 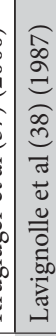 & 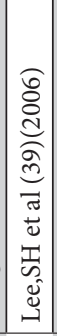 & 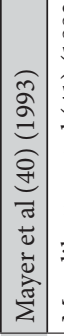 & 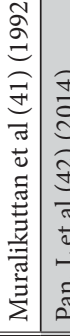 & 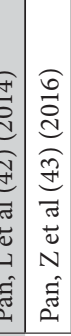 & 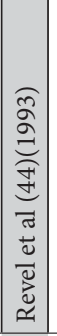 & 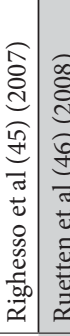 & 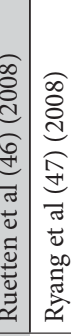 & 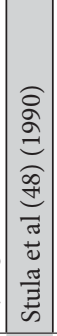 & 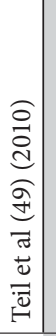 & 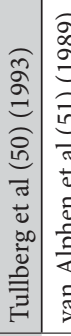 & 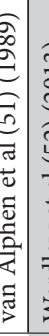 & \\
\hline
\end{tabular}


Random sequence generation (selection bias)

Allocation concealment (selection bias)

Incomplete outcome data (attrition bias)

Selective reporting (reporting bias)

Other bias

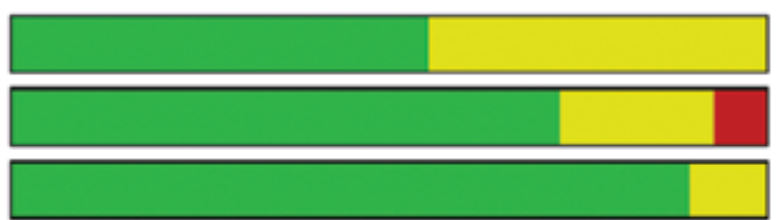

Low risk of bias

\section{Unclear risk of bias}

High risk of bias

Fig. 4. Risk of bias graph. Five items introduced by Cochrane Handbook were considered. Blinding was canceled due to impractical implementation.

Table 2. Results of network meta-analysis for success rate-related outcomes.

\begin{tabular}{|l|c|c|c|c|c|c||}
\hline & SOD & SOMD & CN & MED & PELD & APLD \\
\hline SOD & 1 & & & & & \\
\hline SOMD & $0.77[0.19-2.18]$ & 1 & & & & \\
\hline CN & $0.78[0.44-1.26]$ & $1.44[0.34-3.93]$ & 1 & & & \\
\hline MED & $0.74[0.31-1.62]$ & $1.42[0.26-4.65]$ & $1.03[0.35-2.54]$ & 1 & & 1 \\
\hline PELD & $1.47[0.45-3.70]$ & $2.30[0.69-5.79]$ & $1.99[0.57-5.25]$ & $2.33[0.49-6.85]$ & & 1 \\
\hline APLD & $0.14[0.02-0.55]$ & $0.18[0.04-0.52]$ & $0.19[0.02-0.75]$ & $0.22[0.02-0.91]$ & $0.10[0.01-0.38]$ & \\
\hline PLDD & $0.49[0.15-1.25]$ & $0.79[0.24-1.97]$ & $0.65[0.22-1.58]$ & $0.78[0.15-2.26]$ & $0.41[0.10-1.15]$ & $6.77[0.85-23.45]$ \\
\hline
\end{tabular}

Table 3. Results of network meta-analysis for complication rate-related outcomes.

\begin{tabular}{|l|c|c|c|c|c|c||}
\hline & SOD & SOMD & CN & MED & PELD & APLD \\
\hline SOD & 1 & & & & & \\
\hline SOMD & $1.78[0.65-4.42]$ & 1 & & & & \\
\hline CN & $76.16[0.31-174]$ & $48.21[0.17-119]$ & 1 & & & \\
\hline MED & $2.29[0.98-4.78]$ & $1.43[0.60-2.78]$ & $1.23[0.01-7.88]$ & 1 & & \\
\hline PELD & $0.37[0.09-0.98]$ & $0.23[0.06-0.58]$ & $0.22[0.01-1.32]$ & $0.18[0.04-0.48]$ & 1 & \\
\hline APLD & $172[0.27-401]$ & $119.1[0.16-282]$ & $2.67[0.29-9.08]$ & $92.24[0.12-201]$ & $996.2[0.75-1586]$ & 1 \\
\hline PLDD & $2.52[0.20-10.41]$ & $1.4[0.14-5.21]$ & $1.32[0.01-8.18]$ & $1.17[0.09-4.56]$ & $10.23[0.54-39.94]$ & $2.49[0.01-8.31]$ \\
\hline
\end{tabular}

PELD (OR 4.36, 0.17 - 18.16), PLDD (OR 3.34, 0.22 14.78), MED (OR 1.66, $0.29-5.95$ ), and SOMD (OR 1.15, $0.17-4.29)$. Details pertaining to other comparisons are listed Table 4.

\section{Ranking of Treatments}

In Fig. 5, we summarized the ranking of the 7 surgical interventions in terms of the success rate, complication rate, and reoperation rate. For a higher success rate, PELD might be the best intervention and APLD most likely the worst. For lowering the complication rate, PELD might be the best option and APLD the worst. For lowering the reoperation rate, SOMD might be the best therapy and APLD the worst.

\section{Publication Bias and Sensitivity Analyses}

Publication bias was not assessed because the number of studies was limited $(<10)$. Sensitivity analy- 
Comparison of Surgical Interventions for Lumbar Disc Herniation

Table 4. Results of network meta-analysis for reoperation rate-related outcomes.

\begin{tabular}{|l|c|c|c|c|c|c||}
\hline & SOD & SOMD & CN & MED & PELD & APLD \\
\hline SOD & 1 & & & & & \\
\hline SOMD & $1.15[0.17-4.29]$ & 1 & & & & \\
\hline CN & $15.89[4.07-47.53]$ & $26.78[2.45-105]$ & 1 & & & \\
\hline MED & $1.66[0.29-5.94]$ & $1.97[0.38-6.08]$ & $0.15[0.01-0.61]$ & 1 & & \\
\hline PELD & $4.36[0.17-18.16]$ & $2.91[0.37-11.97]$ & $0.34[0.01-1.55]$ & $2.87[0.16-12.54]$ & 1 & \\
\hline APLD & $46.75[2.78-233]$ & $60.8[3.05-298]$ & $3.22[0.23-15.04]$ & $43.98[1.77-224]$ & $46.07[0.82-246]$ & 1 \\
\hline PLDD & $3.34[0.22-14.78]$ & $3.98[0.26-16.87]$ & $0.30[0.01-1.39]$ & $2.95[0.14-13.69]$ & $3.08[0.06-14.87]$ & $0.25[0.01-1.22]$ \\
\hline
\end{tabular}

ses where 2 studies with a high risk of bias were excluded did not change the result.

\section{Discussion}

\section{Summary of Main Results}

The network meta-analysis provides hierarchies for the success, complication, and reoperation rates of the 7 different in interventions in people with $\mathrm{LDH}$. The meta-analysis indicated that: For increasing the success rate, PELD might be the best surgical intervention and APLD might be the worst. For decreasing the complication rate, PELD might be the best option and APLD might be the worst. For decreasing the reoperation rate, SOMD might be the best therapy and APLD might be the worst.

\section{Strengths and Limitations}

There were some strengths in this article: (1) we used a comprehensive search strategy to minimize the possibility of publication bias; (2) the posterior probabilities of outcomes and SUCRA were used to distinguish the subtle differences among 7 surgical interventions; (3) the article referred to the results of direct and indirect comparisons; and (4) only RCTs that described random sequence generation were included in this article.

However, the results of the review should be interpreted under some limitations. First, both the number of the included studies and the sample size were small, which might affect the outcome. Moreove, $r$ for the success rate, the sample size for APLD was less than 100 . For the complication rate, the sample size for APLD and PLDD were also less than 100. Third, there was substantial heterogeneity due to the inconformity regarding the duration of follow-up. Fourth, for the studies where the success, complication, and reoperation rates were second-

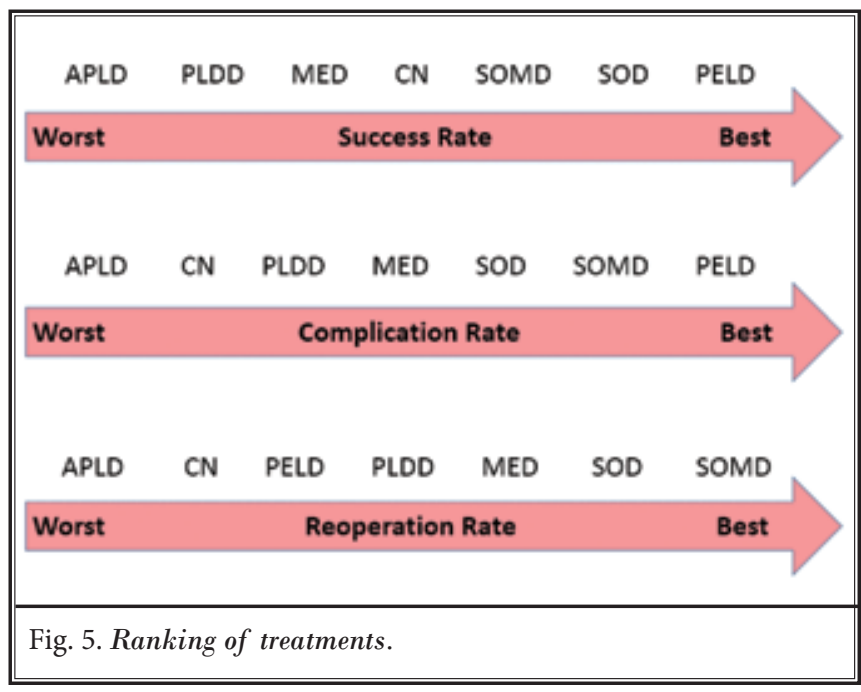

ary outcomes, the number time points was insufficient which might underestimate the outcomes. Fifth, our article used summary data instead of individual patient data, which might lead to the loss of some covariates at the individual patient level. Finally, due to the difference in indications in the included studies, the results might be influenced and need to be carefully applied.

\section{Agreements and Disagreements in the Current Literature}

In prior meta-analyses Rasouli and colleagues (54) showed there were no significant difference between minimally invasive discectomy (PELD/APLD) and SOD/ SOMD for surgical procedure-related complication rates (OR $1.01,95 \% \mathrm{Cl} 0.61$ to $1.66, P=0.97 \mathrm{I}^{2}=33 \%$ ) and reoperation rate (OR $2.13,95 \% \mathrm{Cl} 1.01$ to $4.49, P=0.65$, $\left.\mathrm{I}^{2}=0 \%\right) ;$ He et al (55) compared the complication rates between MED and SOD (OR 1.27, 95\% Cl 0.63 to 2.59, $P=0.22, I^{2}=33 \%$ ), and no significant differences were found. Gibson and Waddell (3) found SOD significantly decreased the reoperation rate compared to CN (OR 
$0.07,95 \% \mathrm{Cl} 0.12$ to $0.08, P<0.001, \mathrm{I}^{2}=0 \%$ ); however, the difference of failure rates between the 2 procedure were insignificant (OR $0.37,95 \% \mathrm{Cl} 0.13$ to $1.05, P=$ $\left.0.006, \mathrm{I}^{2}=78.6 \%\right)$.

Although previous studies have different combinations, the ranking methods are relatively rough, and their included interventions were not comprehensive. Therefore, we performed this network meta-analysis. Overall, our results agree with the previous research and we were able to rank these interventions which the previous reviews did not.

\section{Conclusions}

This meta-analysis provides evidence that PELD might be the best choice to increase the success rate and decrease the complication rate; moreover, SOMD might be the best option to lower the reoperation rate. APLD might lead to the lowest success rate and the highest complication and reoperation rates. Higher quality RCTs and direct head to head trials are needed to confirm these results.

\section{References}

1. Fritsch EW, Heisel J, Rupp S. The failed back surgery syndrome: Reasons, intraoperative findings, and long-term results: A report of 182 operative treatments. Spine (Phila Pa 1976) 1996; 21:626-633.

2. Gibson JN, Cowie JG, Iprenburg M. Transforaminal endoscopic spinal surgery: The future 'gold standard' for discectomy? - A review. Surgeon 2012; 10:290-296.

3. Gibson JN, Waddell G. Surgical interventions for lumbar disc prolapse: Updated Cochrane Review. Spine (Phila Pa 1976) 2007; 32:1735-1747.

4. Cong L, Zhu Y, Tu G. A meta-analysis of endoscopic discectomy versus open discectomy for symptomatic lumbar disk herniation. Eur Spine J 2016; 25:134-143.

5. Blamoutier A. Surgical discectomy for lumbar disc herniation: Surgical techniques. Orthop Traumatol Surg Res 2013; 99:S187-S196.

6. Mixter W, Barr J. Rupture of the intervertebral disc with involvement of the spinal canal. N Engl J Med 1934; 211:205-210.

7. Bokov A, Isrelov A, Skorodumov A, Aleynik A, Simonov A, Mlyavykh S. An analysis of reasons for failed back surgery syndrome and partial results after different types of surgical lumbar nerve root decompression. Pain Physician 2011; 14:545-557.

8. Yasargil MG. Microsurgical operation of herniated lumbar disc. Adv Neurosurg 1977; 4:81-82.

9. Caspar WA. A new surgical procedure for lumbar disc herniation causing less tissue damage through a microsurgical approach. Adv Neurosurg 1977; 4:74-77.
10. Valat JP, Genevay S, Marty M, Rozenberg S, Koes B. Sciatica. Best Pract Res Clin Rheumatol 2010; 24:241-252.

11. Foley MKT. Microendoscopic discectomy. Tech Neurosurg 1997; 3:301-307.

12. Smith L. Enzyme dissolution of the nucleus pulposus in human. JAMA 1964; 187:177-180.

13. Hijikata S, Yamagishi M, Nakayama T, Oomori K. Percutaneous nuclectomy: A new treatment method for lumbar disc herniation. J Toden Hosp 1975; 5:5-13.

14. Onik G, Helms CA, Ginsburg L, Hoaglund FT, Morris J. Percutaneous lumbar diskectomy using a new aspiration probe. AJR Am J Roentgenol 1985; 144:1137-1140.

15. Choy DS, Ascher P, Lammer J, Rothman L, Snowdon J. Percutaneous laser catheter recanalization of carotid arteries in seven cadavers and one patient. AJNR Am J Neuroradiol 1986; 7:1050-1052.

16. Mayer HM, Brock M, Berlien HP, Weber B. Percutaneous endoscopic laser discectomy (PELD). A new surgical technique for non-sequestrated lumbar discs. Acta Neurochir Suppl (Wien) 1992; 54:53-58.

17. Lan T, Chang L, Mn R, Wu L, Yuan YF. Comparative efficacy of Interventional therapies for early-stage hepatocellular carcinoma: A PRISMA-compliant systematic review and network meta-analysis. Medicine (Baltimore) 2016; 95:e3185.

18. Higgins J, Green S. Cochrane Handbook for Systematic Reviews of Interventions Version 5.1.0 [updated March 2011]. The Cochrane Collaboration, 2011.

19. Chaimani A, Higgins JP, Mavridis D, Spyridonos P, Salanti G. Graphical tools for network meta-analysis in STATA. PLoS One 2013; 8:e76654.
20. Sutton A, Ades AE, Cooper N, Abrams $K$. Use of indirect and mixed treatment comparisons for technology assessment. Pharmacoeconomics 2008; 26:753-767.

21. Jansen JP, Crawford B, Bergman G, Stam W. Bayesian meta-analysis of multiple treatment comparisons: An introduction to mixed treatment comparisons. Value Health 2008; 11:956-964.

22. Salanti G, Ades AE, loannidis JP. Graphical methods and numerical summaries for presenting results from multipletreatment meta-analysis: An overview and tutorial. J Clin Epidemiol 2011; 64:163-171.

23. Abrishamkar $S$, Kouchakzadeh $M$, Mirhosseini A, Tabesh H, Rezvani M, Moayednia A, Ganjeifar B, Mahabadi A, Yousefi E, Kooshki AM. Comparison of open surgical discectomy versus plasma-laser nucleoplasty in patients with single lumbar disc herniation. J Res Med Sci 2015; 20:1133-1137.

24. Arts MP, Brand R, van den Akker ME, Koes BW, Bartels RH, Peul WC. Tubular diskectomy vs conventional microdiskectomy for sciatica: A randomized controlled trial. JAMA 2009; 302:149-158.

25. Arts MP, Brand R, van den Akker ME, Koes BW, Bartels RH, Tan WF, Peul WC. Tubular diskectomy vs conventional microdiskectomy for the treatment of lumbar disk herniation: 2-year results of a double-blind randomized controlled trial. Neurosurgery 2011; 69:135-144; discussion 144.

26. Brouwer PA, Brand R, van den Akkervan Marle ME, Jacobs WC, Schenk B, van den Berg-Huijsmans AA, Koes BW, Arts MA, van Buchem MA, Peul WC. Percutaneous laser disc decompression 
versus conventional microdiscectomy in sciatica: A randomized controlled trial. Spine ] 2015; 15:857-865.

27. Burton AK, Tillotson KM, Cleary J. Single-blind randomised controlled trial of chemonucleolysis and manipulation in the treatment of symptomatic lumbar disc herniation. Eur Spine J 2000; 9:202-207.

28. Chatterjee S, Foy PM, Findlay GF. Report of a controlled clinical trial comparing automated percutaneous lumbar discectomy and microdiscectomy in the treatment of contained lumbar disc herniation. Spine (Phila Pa 1976) 1995; 20:734-738.

29. Crawshaw C, Frazer AM, Merriam WF, Mulholland RC, Webb JK. A comparison of surgery and chemonucleolysis in the treatment of sciatica. A prospective randomized trial. Spine (Phila Pa 1976) 1984; 9:195-198.

30. Ejeskar A, Nachemson A, Herberts P, Lysell E, Andersson G, Irstam L, Peterson LE. Surgery versus chemonucleolysis for herniated lumbar discs. A prospective study with random assignment. Clin Orthop Relat Res 1983:236-242.

31. Franke J, Greiner-Perth R, Boehm $H$, Mahlfeld K, Grasshoff H, Allam Y, Awiszus F. Comparison of a minimally invasive procedure versus standard microscopic discotomy: A prospective randomised controlled clinical trial. Eur Spine J 2009; 18:992-1000.

32. Garg B, Nagraja UB, Jayaswal A. Microendoscopic versus open discectomy for lumbar disc herniation: A prospective randomised study. J Orthop Surg (Hong Kong) 2011; 19:30-34.

33. Haines SJ, Jordan N, Boen JR, Nyman JA, Oldridge NB, Lindgren BR. Discectomy strategies for lumbar disc herniation: Results of the LAPDOG trial. J Clin Neurosci 2002; 9:411-417.

34. Hermantin FU, Peters T, Quartararo L, Kambin P. A prospective, randomized study comparing the results of open discectomy with those of video-assisted arthroscopic microdiscectomy. J Bone Joint Surg Am 1999; 81:958-965.

35. Huang TJ, Hsu RW, Li YY, Cheng CC. Less systemic cytokine response in patients following microendoscopic versus open lumbar discectomy. J Orthop Res 2005; 23:406-411.

36. Hussein M, Abdeldayem A, Mattar MM. Surgical technique and effectiveness of microendoscopic discectomy for large uncontained lumbar disc herniations: A prospective, randomized, controlled study with 8 years of follow-up. Eur Spine J 2014; 23:1992-1999.

37. Krugluger J, Knahr K. Chemonucleolysis and automated percutaneous discectomy: A prospective randomized comparison. Int Orthop 2000; 24:167-169.

38. Lavignolle B, Vital JM, Baulny D, Grenier F, Castagnera L. [Comparative study of surgery and chemonucleolysis in the treatment of sciatica caused by a herniated disk]. Acta Orthop Belg 1987; 53:244-249.

39. Lee SH, Chung SE, Ahn Y, Kim TH, Park JY, Shin SW. Comparative radiologic evaluation of percutaneous endoscopic lumbar discectomy and open microdiscectomy: A matched cohort analysis. Mt Sinai] Med 2006; 73:795-801.

40. Mayer HM, Brock M. Percutaneous endoscopic discectomy: Surgical technique and preliminary results compared to microsurgical discectomy. J Neurosurg 1993; 78:216-225.

41. Muralikuttan KP, Hamilton A, Kernohan WG, Mollan RA, Adair IV. A prospective randomized trial of chemonucleolysis and conventional disc surgery in single level lumbar disc herniation. Spine (Phila $\mathrm{Pa} \mathrm{1976)} \mathrm{1992;} \mathrm{17:381-387.}$

42. Pan L, Zhang P, Yin Q. Comparison of tissue damages caused by endoscopic lumbar discectomy and traditional lumbar discectomy: A randomised controlled trial. Int J Surg 2014; 12:534-537.

43. Pan Z, Ha Y, Yi S, Cao K. Efficacy of Transforaminal Endoscopic Spine System (TESSYS) technique in treating lumbar disc herniation. Med Sci Monit 2016; 22:530-539.

44. Revel M, Payan C, Vallee C, Laredo JD, Lassale B, Roux C, Carter H, Salomon C, Delmas E, Roucoules J. Automated percutaneous lumbar discectomy versus chemonucleolysis in the treatment of sciatica. A randomized multicenter trial. Spine (Phila Pa 1976) 1993; 18:1-7.

45. Righesso O, Falavigna A, Avanzi O. Comparison of open discectomy with microendoscopic discectomy in lumbar disc herniations: Results of a randomized controlled trial. Neurosurgery 2007; 61:545-549; discussion 549.

46. Ruetten S, Komp M, Merk H, Godolias G. Full-endoscopic interlaminar and transforaminal lumbar discectomy versus conventional microsurgical tech- nique: A prospective, randomized, controlled study. Spine (Phila Pa 1976) 2008; 33:931-939.

47. Ryang YM, Oertel MF, Mayfrank L, Gilsbach JM, Rohde V. Standard open microdiscectomy versus minimal access trocar microdiscectomy: Results of a prospective randomized study. Neurosurgery 2008; 62:174-181; discussion 181-182.

48. Stula D. [Chemonucleolysis with chymopapain in lumbar disk hernia. Randomized comparative study with operated patients]. Neurochirurgia (Stuttg) 1990; 33:169-172.

49. Teli M, Lovi A, Brayda-Bruno M, Zagra A, Corriero A, Giudici F, Minoia L. Higher risk of dural tears and recurrent herniation with lumbar micro-endoscopic discectomy. Eur Spine ] 2010; 19:443-450.

50. Tullberg T, Isacson J, Weidenhielm L. Does microscopic removal of lumbar disc herniation lead to better results than the standard procedure? Results of a one-year randomized study. Spine (Phila Pa 1976) 1993; 18:24-27.

51. van Alphen HA, Braakman R, Bezemer PD, Broere G, Berfelo MW. Chemonucleolysis versus discectomy: A randomized multicenter trial. J Neurosurg 1989; 70:869-875.

52. Wardlaw D, Rithchie IK, Sabboubeh AF, Vavdha M, Downing M, Eastmond CJ. Prospective randomized trial of chemonucleolysis compared with surgery for soft disc herniation with 1-year, intermediate, and long-term outcome: Part II: The radiological outcome. Spine (Phila Pa 1976) 2013; 38:E1058-E1064.

53. Wardlaw D, Rithchie IK, Sabboubeh AF, Vavdha M, Eastmond CJ. Prospective randomized trial of chemonucleolysis compared with surgery for soft disc herniation with 1-year, intermediate, and long-term outcome: Part I: The clinical outcome. Spine (Phila Pa 1976) 2013; 38:E1051-E1057.

54. Rasouli MR, Rahimi-Movaghar V, Shokraneh F, Moradi-Lakeh M, Chou R. Minimally invasive discectomy versus microdiscectomy/open discectomy for symptomatic lumbar disc herniation. Cochrane Database Syst Rev 2014; 9:Cdo10328.

55. He J, Xiao S, Wu Z, Yuan Z. Microendoscopic discectomy versus open discectomy for lumbar disc herniation: A metaanalysis. Eur Spine J 2016; 25:1373-1381. 
